\title{
Challenging the estimation of cortical activity from MEG with simulated fMRI-constrained retinotopic maps
}

\author{
Alexandre Gramfort, Benoit Cottereau, Maureen Clerc, Bertrand Thirion, Sylvain Baillet
}

\begin{abstract}
Detection of activity from the primary visual cortex is a difficult challenge to magneto-encephalography (MEG) source imaging techniques: the geometry of the visual cortex is intricate, with structured visual field maps extending deeper along the calcarine fissure. This questions the very sensitivity of MEG to the corresponding neural responses of visual stimuli and the usage of MEG source imaging for innovative retinotopic explorations. In this context, we compare two imaging models of MEG generators in realistic simulations of activations within the visual cortex. Localization and spatial extent of neural activity in the visual cortex were extracted from retinotopic maps obtained in fMRI. We prove that the suggested approaches are robust and succeed in accurately recovering the activation patterns with satisfactory match with fMRI results. These results suggest that fast retinotopic exploration of the visual cortex could be obtained from MEG as a complementary alternative to more standard fMRI approaches. The excellent time resolution of MEG imaging further opens interesting perspectives on the temporal and spectral processes sustained by the human visual system.
\end{abstract}

\section{INTRODUCTION}

Neural activity is sustained by intracellular - primary - currents that loop within the brain volume owing to secondary - or volumic - extracellular currents [2]. Magnetoencephalography (MEG) essentially detects the magnetic fields produced by neocortical pyramidal neurons, whose combined activity at the scale of neural assemblies can be efficiently modeled by an equivalent current dipole (ECD) oriented perpendicularly to the cortical surface [8]. Imaging approaches to the estimation of the neural generators of MEG are based on the distribution of at least 10,000 ECDs on a tessellated model of the cortical envelope.

By modeling the cortical currents with such a dense surfacic dipole field, distributed dipole models yield an interesting imaging alternative to the more conventional localization approaches based on non-linear dipole fits and other scanning techniques. Image models for MEG generators also suggest that the very spatial extension of neural activations could be extracted from the data. Reliable estimation of this parameter would advantageously complement basic measures of brain

A. Gramfort and M. Clerc are with the Odyssée Project Team (ENPC - ENS - INRIA) alexandre.gramfortesophia.inria.fr maureen.clercesophia.inria.fr

B. Cottereau is with the ESME-Sudria College of Engineering, Ivry, France cottereaudesme. fr

B. Thirion is with the INRIA Futurs, Neurospin bertrand.thirioneinria.fr

B. Cottereau and S. Baillet are with Cognitive Neuroscience \& Brain Imaging Laboratory, CNRS UPR 640 LENA, MEG Centre, University Pierre \& Marie CURIE Paris6, Paris, France benoit.cottereaulchups.jussieu.fr sylvain.baillet@chups.jussieu.fr responses in multiple experimental paradigms. One canonical example is vision, where brain responses to visual stimuli have been extensively explored in animal models and humans. fMRI has revealed a number of keys in the retinotopic organization of visual cortices where neural responses are structured into visual field maps [10]. The intricate functional and anatomical organization of the human visual system is a challenge to EEG and MEG imaging [4], whose spatial resolution is usually considered on the order of the centimeter and rapidly degrades with distance to sensors. Here we report on the performances of recent forward and inverse MEG image models in the difficult context of neural responses within the visual cortex. We compare the images of neural activations obtained from MEG to the regions identified from fMRI, a technique known for its excellent spatial resolution.

\section{Methods}

\section{A. Forward and inverse modeling in $M E G$}

Solving the direct problem in EEG consists in computing the electrical potential $V(r)$ given the conductivity $\sigma(r)$ in the volume $\Omega$ and the primary current $J^{p}(r)$ within the cortex. The Maxwell equations with the quasi-static approximation give the following equation :

$$
\nabla \cdot(\sigma \nabla V)=\nabla \cdot J^{p} \text { in } \Omega
$$

The forward problem in MEG computes the magnetic field $\mathbf{B}(r)$ given the primary current $J^{p}(r)$, the conductivity of volumes $\sigma(r)$ and the potential $V(r)$ using the Biot-Savart equation. The inverse problem in MEG aims at evaluating the primary currents on the cortex from measurements on the MEG sensors.

\section{B. Symmetric BEM solution with variational energy-based inverse problem resolution}

Boundary element methods (BEM) belong to the general category of finite element methods which are commonly used to solve partial differential equations over complex domains. The BEM consists in substituting computation of integrals over domains with integrals over surfaces. The symmetric BEM approach [7] samples both the potential $V$ and the normal currents on the surfaces $\sigma \partial_{\mathbf{n}} V$.

The geometry of the head model consists of 4 nested homogenous domains ('brain' including cerebrospinal fluid (CSF), 'skull', 'skin' and 'air') and therefore 3 nested interfaces. The brain interface separates the CSF and the inner skull surface, the skull envelope corresponds to the 


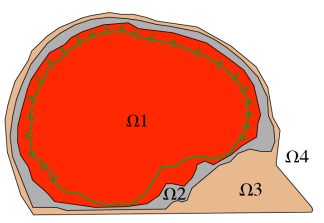

(a)

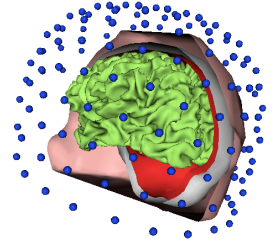

(b)
Fig. 1. (a) Schematic representation of the head model with 3 nested interfaces and the cortex covered with dipoles normally distributed on the surface $\left(\Omega_{1}=\right.$ Brain with cerebrospinal fluid, $\Omega_{2}=$ Skull, $\Omega_{3}=$ Skin and $\Omega_{4}=$ Air) (b) Realistic head model obtained by segmenting a T1 MRI scan (about 500 vertices per interface. The cortical envelope is a triangulation with 35,000 vertices)

outer skull surface and the skin is the outer surface of the scalp (cf. Fig. 1).

The method leads to a linear formulation of the problem:

$$
\mathbf{M}=\mathbf{H S}
$$

where $\mathbf{H}$ is the lead field matrix relating the sources to the measurements, $\mathrm{M}$ is a vector of $N_{m}$ instantaneous measurements on the MEG sensor array at a given time frame, and $\mathbf{S}$ is a vector of corresponding elementary source amplitudes from the distributed cortical image model.

The MEG inverse problem is severely ill-posed and requires therefore complementary assumptions on the expected solution. Such priors generally enforce some regularizing smoothness in the distribution of source amplitudes. Formally, this property is translated in the estimation process through the minimization of an energy functional $E(\mathbf{S})$ with two terms: $E_{\text {data }}(\mathbf{S})$ that balances between the fit of the forward model from $S$ with the measurements on the MEG sensors, and $E_{\text {smooth }}(\mathbf{S})$, a term that imposes the distribution of source amplitudes to be spatially smooth:

$$
E(\mathbf{S})=E_{\text {data }}(\mathbf{S})+\lambda E_{\text {smooth }}(\mathbf{S})
$$

where $E_{\text {data }}(\mathbf{S})=\|\mathbf{M}-\mathbf{H S}\|^{2}$ and $\lambda$, a positive scalar, controls for the smoothness of source amplitudes. Here we suggest the following two image models :

Minimum norm of the gradient (HEAT) : $E_{\text {smooth }}=\|\nabla \mathbf{S}\|^{2}$ Total variation (TV) $\quad: E_{\text {smooth }}=\|\nabla \mathbf{S}\|$

where $\nabla$ stands for the gradient on the cortical manifold (see [1] for a complete description of the associated optimization approaches).

Optimization of the TV model has been well studied in the image processing community for denoising purposes. Results show that blurring of estimated source intensities is considerably reduced in MEG using this model. The associated optimization functional however is non convex and may require a considerable number iterations before it reaches convergence, thereby impeding its practical usage.

\section{Alternative multiresolution approach}

As over 10,000 elementary sources are necessary to properly sample the cortical anatomy, estimating $\mathbf{S}$ from $\mathbf{M}$

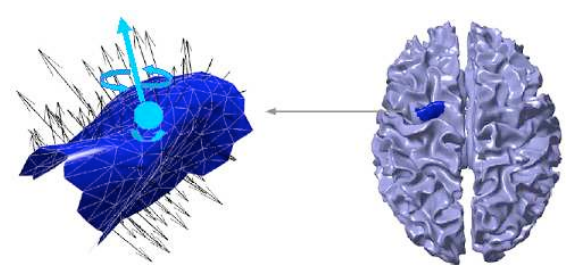

Fig. 2. Illustration of a $300 \mathrm{~mm}^{2}$ cortical patch. Moments of current quadrupolar expansion are obtained from the elementary dipole moments supporting the patch geometry, expanded about the parcel centroid.

is extremely underdetermined, thereby aggravating the illposedness of the inverse estimation. We therefore suggest a multiresolution image model to circumvent this problem. At each spatial resolution $k$, the image model $\mathcal{M}_{k}$ is segmented into $N_{c}$ clusters $\mathcal{C}_{j}^{k}$ of elementary sources:

$$
\mathcal{M}_{k}=\left\{\mathcal{C}_{j}^{k}, j \in\left[1, N_{c}\right]\right\}
$$

Some sources are eliminated at each resolution $k$ while others are brought to the next image resolution $k+1$ and distributed in a new image model through the following steps:

1) Design of the piecewise image model $\mathcal{M}_{k+1}$ at resolution $k+1$ from the elementary sources that survived previous eliminatory procedure of Step 3;

2) Compact parametric modeling of regional neural activity from each elementary cluster $\mathcal{C}_{j}^{k+1}$ in $\mathcal{M}_{k+1}$;

3) Model selection: eliminate the least-significant source cluster from $\mathcal{M}_{k}$ and loop back to Step 1.

We now briefly detail the technical aspects of the modelisation and selection steps (see [3] for a full description).

At each resolution $k$, the available cortical sources are clustered in $N_{c}$ patches $\mathcal{C}_{j}^{k}$ of similar surface area. Jerbi et al. showed in [5] that the current quadrupolar expansion was an adequate model for extended sources $\left(>5 \mathrm{~cm}^{2}\right)$ with only 7 moment parameters accounting for the cortical activity generally supported by about 100 elementary dipoles in conventional imaging models (see Fig. 2). At resolution $k$, the activity of cluster $\mathcal{C}_{j}^{k}$ is modeled up to its quadrupolar expansion about its geometrical centroid. (2) becomes:

$$
\mathbf{M}=\overline{\mathbf{H}}_{k} \overline{\mathbf{Q}}_{k}
$$

where $\overline{\mathbf{H}}_{k}$ is the $\left(N_{m} \times N_{p}\right)$ associated gain matrix up to quadrupolar moments. Note that as $N_{p}=7 N_{c}$, we can enforce the problem to be overdetermined by assuming $N_{c} \leq N_{m} / 20$ at each spatial resolution $k$.

In order to successively eliminate the least-significant cluster in the source model, we compute the Generalized Cross-Validation error (GCV-error) $\epsilon_{j}^{k}$ for $N_{c}$ submodels indexed by $j$ consisting of all clusters in $\mathcal{M}_{k}$ except $\mathcal{C}_{j}^{k}$. In the context of a Weighted Minimum-Norm Estimate (WMNE) of the quadrupolar moments controlled by a regularisation 


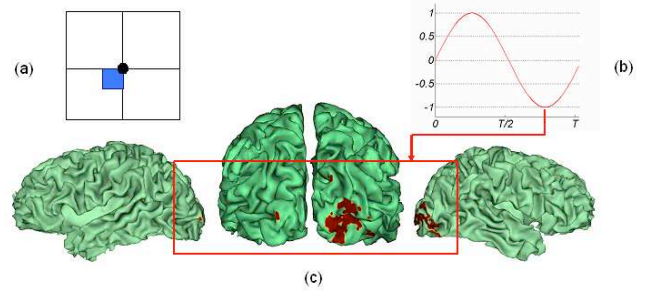

Fig. 3. The stimulus presented in the visual field in (a) yields activity identified from fMRI (c). Simulated MEG data consists in the corresponding cortical locations supporting a sinewave time course (b) and associated forward model.

parameter $\lambda$, this error writes analytically [9] :

$$
\epsilon_{j}^{k}=\frac{\left\|\left(\overline{\mathbf{H}}_{k \mid j} \overline{\mathbf{H}}_{k \mid j}^{t}+\lambda \overline{\mathbf{C}}_{k \mid j}^{-1}\right)^{-1} \mathbf{M}\right\|}{\operatorname{Trace}\left(\left(\overline{\mathbf{H}}_{k \mid j} \overline{\mathbf{H}}_{k \mid j}^{t}+\lambda \overline{\mathbf{C}}_{k \mid j}^{-1}\right)^{-1}\right)}
$$

where $\overline{\mathbf{C}}_{k \mid j}^{-1}$ is a spatial covariance prior on quadrupolar moments of clusters $M_{k \mid j}$ at resolution $k$ (see [3]). The cluster associated with the smallest GCV-error $\mathcal{C}_{j_{0}}^{k}$ is supposed to be the least significant and is removed at resolution $k+1$.

At the end of this exhaustive procedure when all elementary dipoles have been removed from the image model, the best model in the GCV-error sense is selected retrospectively. As the initial cortical parcellation at $k=0$ is arbitrary and coarse, the entire process is restarted $L$ times. A weighted summation of the individual GCV errors across the $L$ best models yields a so-called multiresolution clustering (MRC) frequency map of dipole amplitudes.

\section{Simulation Study}

\section{A. Description}

MEG data from source distributions of neural currents were simulated from retinotopic maps revealed by fMRI [11]. Portions of the maps corresponding to a particular region of the visual field were selected. Neural activations within these regions were simulated with a sine wave (cf. Fig. 3). The resulting activities on the MEG sensors were computed using the BEM forward model described in Section II-B. Locations and orientations of the MEG sensors were those of the OMEGA-151 system from VSM MedTech (Coquitlam, $B C$, Canada) and consisted of 151 axial gradiometers. The cortical envelope containing about 35,000 vertices was extracted from a T1 MRI with BrainVISA ${ }^{1}$. Two experimental conditions were tested: one noiseless (cf. Fig. 4-a), and one with strong additive white noise $(S N R=1$ in variance ratio), cf. Fig. 5-a. Sections III-B and III-C detail the results from the evaluated methods for the simulation illustrated Fig. 3. Section III-D provides a global analysis of the performances from the simulated data sets.

\begin{tabular}{|c|c|c|c|}
\cline { 2 - 4 } \multicolumn{1}{c|}{} & Direct problem & \multicolumn{2}{c|}{ Inverse problem } \\
\hline \multirow{2}{*}{ BEM } & Computation of $\mathbf{H}_{B E M}$ & HEAT & TV \\
\cline { 2 - 4 } & $3 \mathrm{~h}$ & $9.5 \mathrm{~s}$ & 6.1s (79 iterations) \\
\hline \multirow{2}{*}{ MRC } & Computation of $\mathbf{H}_{M R C}$ & \multicolumn{2}{|c|}{ Frequency Map } \\
\cline { 2 - 4 } & $10 \mathrm{~s}$ & \multicolumn{3}{|c}{30 min. (30 Trials) } \\
\hline
\end{tabular}

TABLE I

COMPUTATION TIMES REQUIRED BY THE FORWARD AND THE INVERSE PROBLEMS (1 TIME FRAME)

\section{B. Results Method 1: Symmetric BEM with variational ap- proach}

In the absence of noise, we can observe (cf. Fig. 4-c and 4-d) that both smoothing methods succeed in localizing the peak of activity. As expected, the solutions of TV and HEAT provide contrasted reconstructions. With noisy data (cf. Fig. 5) the TV solution appears to be more robust than the HEAT solution. This is confirmed by the ROC study in III-D.

Computation times are presented in Table I. The computation has been performed on an Intel ${ }^{\mathrm{TM}}$ Core 2 Duo 2.33 $\mathrm{GHz}$ processor with $2 \mathrm{~GB}$ of RAM. The ATLAS Library was used for linear algebra operations.

\section{Results Method 2: Multiresolution approach}

Data processing, forward modelling and visualization were obtained with the BrainStorm toolbox ${ }^{2}$ using a spherical head geometry. Fig. 4 and Fig. 5-e present the MRC frequency map obtained from the simulated activity given by Fig. 3-a (see Fig. 4 and Fig. 5-a and b for the corresponding signals and topographies) with $L=30$ resolution descent trials. Accuracy is satisfactory even with noisy data as confirmed by the AUC estimation in Section III-D.

These results were computed on a Pentium $\AA 43 \mathrm{GHz}$ with $1 \mathrm{~GB}$ of RAM. It took about one minute to compute one trial of the algorithm, leading to a global computing time of 30 minutes to get the MRC frequency map (cf. Table I).

\section{ROC Study}

The forward and inverse models were further estimated for additional stimulation configurations (4 quadrants and 3 eccentricities within a quadrant) and Receiver Operating Curves (ROC) were computed for each stimulus. These curves are obtained by plotting $\mathcal{S}_{e}(\beta)$ against $1-\mathcal{S}_{p}(\beta)$ with:

$$
\begin{aligned}
\mathcal{S}_{e}(\beta) & =\frac{T P(\beta)}{T P(\beta)+F N(\beta)} \\
\mathcal{S}_{p}(\beta) & =\frac{T N(\beta)}{T N(\beta)+F P(\beta)},
\end{aligned}
$$

where $T P, F P, T N$ and $F N$ are the true positive, false positive, true negative and false negative detection rates for a cutoff value of $\beta$ (see [6] for a full description). The Area

${ }^{2}$ http://neuroimage.usc.edu/brainstorm 


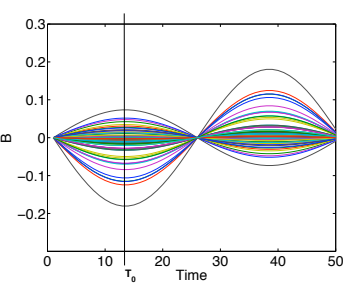

(a) Forward problem

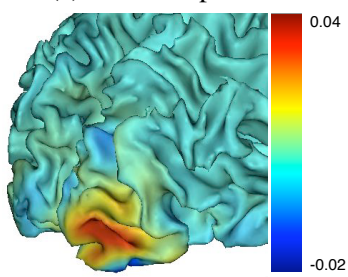

(c) BEM: HEAT

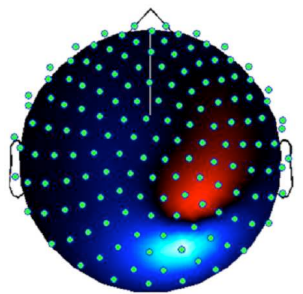

(b) Topography on MEG Sensors

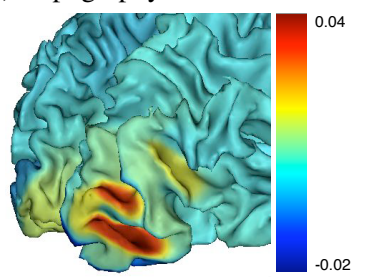

(d) BEM: TV

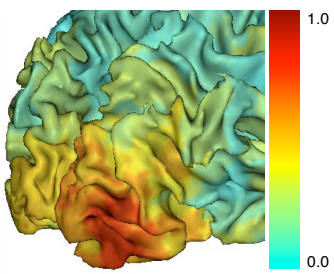

(e) MRC frequency map

Fig. 4. Results with clean direct problem at time $T_{0}$.

\begin{tabular}{|l|c|c|c|c|}
\cline { 3 - 5 } \multicolumn{2}{c|}{} & HEAT & TV & MRC \\
\hline \multirow{2}{*}{ Quadrants } & No Noise & 0.9343 & 0.8818 & 0.8607 \\
& SNR = 1 & 0.8104 & 0.8620 & 0.7722 \\
\hline \multirow{2}{*}{ Eccentricities } & No Noise & 0.9353 & 0.8632 & 0.9038 \\
& SNR = 1 & 0.7837 & 0.8349 & 0.8486 \\
\hline
\end{tabular}

TABLE II

RESULTS OF THE ROC STUDY OBTAINED BY SIMULATIONS ON THE 4 QUADRANTS AND FOR 3 DIFFERENT ECCENTRICITIES

Under the Curve (AUC) provides a global quality index of the estimate: the closer to 1, the better the estimation. Table II displays mean AUC values obtained for the methods under evaluation.

Results show that all three methods give good results on noiseless conditions, but also provide satisfactory reconstructions even with a significant amount of noise. We also notice that, in these experiments, TV smoothing is the most robust to noise.

\section{CONCLUSIONS AND FUTURE WORK}

We have confronted results of two significantly different approaches and discussed results obtained from both image models. By running multiple experiments on real retinotopic fMRI data, we proved that distributed methods in MEG are of interest for localizing spatially extended activations especially in the visual cortex.

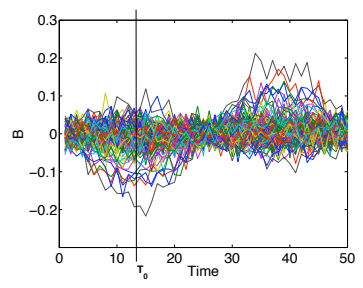

(a) Forward problem

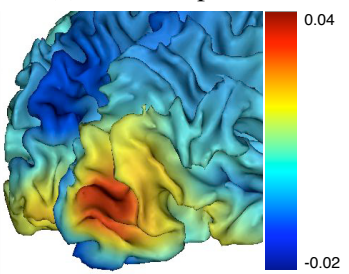

(c) BEM: HEAT

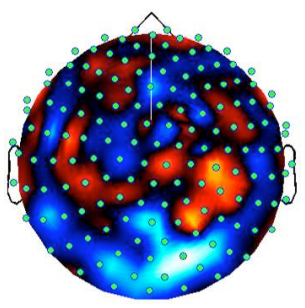

(b) Topography on MEG Sensors

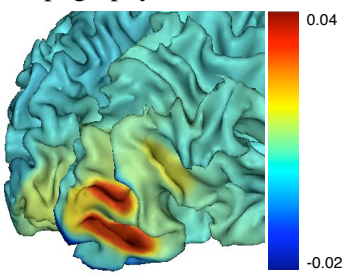

(d) BEM: TV

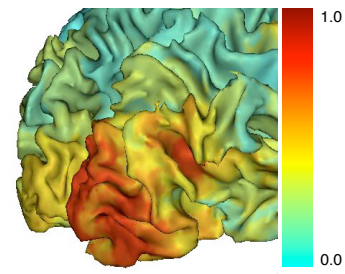

(e) MRC frequency map

Fig. 5. Results with noisy direct problem $(S N R=1)$ at time $T_{0}$.

The natural evolution of this work is to setup an experimental protocol using visual stimuli to evaluate such methods on real MEG measurements.

\section{REFERENCES}

[1] G. Adde, M. Clerc, and R. Keriven. Imaging methods for MEG/EEG inverse problem. In NFSI, 2005.

[2] S. Baillet, J.C. Mosher, and R.M. Leahy. Electromagnetic brain mapping. IEEE Sig. Proc. Mag., 18(6):14-30, 2001.

[3] B. Cottereau, K. Jerbi, and S. Baillet. Multiresolution imaging of neural currents from MEG data using an explicit piecewise image model. In IEEE ISBI, 2006.

[4] Chang-Hwan Im, Arvind Guruirajan, Nanyin Zhang, Wei Chen, and Bin He. Spatial resolution of EEG cortical source imaging revealed by localization of retinotopic organization in human primary visual cortex. Journal of Neuroscience Methods, 161:142-154, 2007.

[5] K. Jerbi, J.C. Mosher, S. Baillet, and R.M. Leahy. On MEG forward modelling using multipolar expansions. Phys. Med. Biol., 47:523-555, 2002.

[6] S. M. Kay. Fundamentals of Statistical Signal Processing: Estimation Theory. Prentice Hall, Englewood Cliffs, NJ, 1993.

[7] J. Kybic, M. Clerc, O. Faugeras, R. Keriven, and T. Papadopoulo. Generalized head models for MEG/EEG: boundary element method beyond nested volumes. Phys. Med. Biol., 51:1333-1346, 2006.

[8] Shingo Murakami and Yoshio Okada. Contributions of Principal Neocortical Neurons to Magnetoencephalography (MEG) and Electroencephalography (EEG) Signals. J Physiol (Lond), 2006.

[9] N. Nguyen, P. Milanfar, and G. Golub. A computationally efficient superresolution image reconstruction algorithm. IEEE Trans. Imag. Proc., 10:57-558, 2001.

[10] B. A. Wandell. Computational neuroimaging of human visual cortex. Annu Rev Neurosci, 22:145-173, 1999.

[11] N. Wotawa, B. Thirion, E. Castet, J.-L. Anton, and O. Faugeras. Human retinotopic mapping using fMRI. INRIA Research Report 5472, 2005. 